University of Wollongong

Research Online

Faculty of Engineering and Information

Faculty of Engineering and Information

Sciences - Papers: Part A

Sciences

$1-1-2016$

\title{
Rapid transformation of hexagonal to cubic silicon carbide (sic) by electric discharge assisted mechanical milling
}

lis Siti Aisyah

University of Wollongong, isa105@uowmail.edu.au

Monika Wyszomirska

University of Wollongong, mw966@uowmail.edu.au

Andrzej Calka

University of Wollongong, acalka@uow.edu.au

David Wexler

University of Wollongong, davidw@uow.edu.au

Follow this and additional works at: https://ro.uow.edu.au/eispapers

Part of the Engineering Commons, and the Science and Technology Studies Commons

Research Online is the open access institutional repository for the University of Wollongong. For further information contact the UOW Library: research-pubs@uow.edu.au 


\title{
Rapid transformation of hexagonal to cubic silicon carbide (sic) by electric discharge assisted mechanical milling
}

\author{
Abstract \\ Silicon carbide powder was successfully transformed from hexagonal SiC into cubic SiC using the electric \\ discharge assisted mechanical milling (EDAMM) method. The milling process was conducted in nitrogen \\ plasma at atmospheric pressure. The effects of pulsed alternating current (AC) and direct current (DC) \\ discharge on product formation were investigated. Products were characterized by X-ray diffractometry, \\ scanning electron microscopy, transmission electron microscopy and nanohardness. It was found that \\ hexagonal SiC can be transformed into cubic SiC under EDAMM processing, near complete \\ transformation occurring within 5 min when applying AC mode electrical pulses, and within 10 min when \\ applying DC mode discharges.
}

\section{Keywords}

mechanical, assisted, discharge, electric, sic, carbide, silicon, milling, cubic, rapid, hexagonal, transformation

\section{Disciplines}

Engineering | Science and Technology Studies

\section{Publication Details}

Aisyah, I. S., Wyszomirska, M., Calka, A. \& Wexler, D. (2016). Rapid transformation of hexagonal to cubic silicon carbide (sic) by electric discharge assisted mechanical milling. Plasma Chemistry and Plasma Processing, 36 1177-1186. 


\title{
Rapid Transformation of Hexagonal to Cubic Silicon Carbide (SiC) by Electric Discharge Assisted Mechanical Milling
}

\author{
I.S. Aisyah ${ }^{1}$, M.Wyszomirska ${ }^{1}$, A. Calka ${ }^{1}$, D. Wexler ${ }^{1}$,
}

Faculty of Engineering, University of Wollongong, NSW, Australia

*Corresponding author:

Monika Wyszomirska, email: mw966@uowmail.edu.au

1. EIS Faculty, Wollongong University, Northfields Ave, Wollongong, NSW 2522, Australia

Tel: +61 415743256; Fax: +61-2-42213112

\begin{abstract}
Silicon Carbide powder was successfully transformed from hexagonal $\mathrm{SiC}$ into cubic $\mathrm{SiC}$ using the Electric Discharge Assisted Mechanical Milling (EDAMM) method. The milling process was conducted in nitrogen plasma at atmospheric pressure. The effects of pulsed Alternating Current (AC) and Direct Current (DC) discharge on product formation were investigated. Products were characterized by X-ray Diffractometry (XRD), Scanning Electron Microscopy (SEM), Transmission Electron Microscopy (TEM) and Nanohardness. It was found that hexagonal $\mathrm{SiC}$ can be transformed into cubic $\mathrm{SiC}$ under EDAMM processing, near complete transformation occurring within 5 min when applying AC mode electrical pulses, and within 10 min when applying DC mode discharges.
\end{abstract}

\section{Keywords: silicon carbide, electric discharge assisted mechanical milling, spark plasma.}

\section{Introduction}

Silicon Carbide is a well-known refractory structural ceramic with a history going back to the $19^{\text {th }}$ century. It is widely used in industrial applications as abrasives, cutting tool elements, heating elements, thermal barriers for aeronautic or aerospace applications. It is relatively inexpensive with high hardness, high strength, low bulk density, good wear, corrosion and oxidation resistance and excellent thermal stability. These properties, combined with its high breakdown field and wide band gap make it a promising semiconductor for high temperature, high frequency and next generation high power devices applications [1]. Specific crystallographic polymorphs of $\mathrm{SiC}$ powder are synthesised via a range of methods, often requiring long processing times or complex processing procedures. These include; mechanochemical processing and hot consolidation [1,2], chemical vapour deposition [3], carbothermic reduction [4,5], carbothermal reduction [6], aerosol assisted synthesis and DC thermal plasma synthesis [7]. Cubic $\mathrm{SiC}$ is attractive as it exhibits high thermal conductivity and refractoriness, low thermal expansion and outstanding abrasion/erosion resistance. It also has a low background current, good electrochemical activity and can be n- or p-doped reaching up to high electrical conductivities [8]. The properties of the final product vary depending on the purity of the ceramic and its crystallographic structure. Within the most commonly used SiC crystallographic structures we can distinguish between $\alpha$ phases corresponding to the hexagonal structures and $\beta$ phases corresponding to the cubic phase. Commonly used nomenclature to further distinguish in between the polymorphic structures of these phases is $4 \mathrm{H}$ and $6 \mathrm{H} \mathrm{SiC}$ for hexagonal and $3 \mathrm{C}$ for the cubic ones.

Silicon carbide experiences a hexagonal/cubic crystal structure transition which was proven in late 60's [9] to be triggered by not only high temperature (in the range of 2000- 
$2300^{\circ} \mathrm{C}$ ) and pressure but also impurities and defects. The literature review reveals that the study concentrated mainly on the cubic to hexagonal transformation rather than the reverse one and so the experimental results in this work will be able to complement the knowledge on this transition in pulsed plasma conditions which hasn't been reported before. Research in this area of is even more important as the thermal stabilities of the $6 \mathrm{H}$ and $3 \mathrm{C} \mathrm{SiC}$ polymorphs suggest that heating will naturally promote formation of the hexagonal crystal structure which is stable above $1700^{\circ} \mathrm{C}$. Reverse transformation on the other hand is relatively hard to achieve as the structure has to rearrange itself from an already stable form and cannot be achieved by simply decreasing the temperature of the process.

Taking into account solely the $6 \mathrm{H} \rightarrow 3 \mathrm{C}$ transformation there are only a few records on the successful reaction. Turan and Knowles [10] studied the influence of the secondary phase on the hexagonal-cubic so-called "reverse" phase transformations, in $\mathrm{Si}_{3} \mathrm{~N}_{4}$ reinforced $6 \mathrm{H}-\mathrm{SiC}$ composite. The phase transformation occurred to a low extent even though the Hot Isostatic Pressing was used at the temperature of $2100^{\circ} \mathrm{C}$ and pressure of $200 \mathrm{MPa}$ in argon. It was then recently reported by Limeng et al. [11] that this phase transformation can occur fast and to a greater extent when the additional $\mathrm{Al}_{2} \mathrm{O}_{3}, \mathrm{Y}_{2} \mathrm{O}_{3}$ and $\mathrm{AlN}$ are included in the composite. Spark Plasma Sintering was able to facilitate this transformation in a much lower temperature of $1700^{\circ} \mathrm{C}$, pressure increasing from 5 to $30 \mathrm{MPa}$ in time as short as 30s. Additionally, the Liquid Phase Sintered method reveals, that nitrogen atmosphere together with the $\mathrm{Al}_{2} \mathrm{O}_{3}$ and $\mathrm{Y}_{2} \mathrm{O}_{3}$ additives suppresses the $6 \mathrm{H} \rightarrow 3 \mathrm{C}$ transformation which otherwise is completed in argon atmosphere. More importantly though the authors state that the nitrogen atmosphere leads to its incorporation in the intergranular phase which increases the hardness of the $\mathrm{SiC}$ in higher temperatures [11]. For the above reasons, the reasonable step taken was to use the EDAMM process to attempt on the phase transition in the nitrogen atmosphere.

It should be noted that none of the above methods were previously reported to be successful in that kind of phase transformation without any additives. Pure $\mathrm{SiC}$ powder processed by the High Energy Ball Milling (mechanosynthesis) method was only able to produce the $3 \mathrm{C}-\mathrm{SiC}$ and no phase transformation was recorded [12]. No $\beta \rightarrow \alpha$ transformation was also noted in [13] where ball milled powder was subjected to Spark Plasma Sintering process in the temperature range of $1650-2220^{\circ} \mathrm{C}$ and up to $150 \mathrm{MPa}$ pressure.

Here, we present Electric Discharge Assisted Mechanical Milling (EDAMM) as a synthesis and processing method demonstrated to promote rapid transformations allowing reaction between freshly fractured particles and ions associated with the generated dusty plasma [15]. In comparison to the previously described methods, EDAMM holds an advantage of promoting the interface rather than the diffusional transformation. Because of that fact, when the pressure as low as $90 \mathrm{kPa}$ is applied and only localised temperature occurs with additional milling, the phase transformations can occur in much lower temperatures. We decided to investigate EDAMM processing of hexagonal $\mathrm{SiC}$ in nitrogen rich plasma to determine if the method could be applied to promote the transformation to refractory cubic SiC. The investigation of phase transformations in hexagonal $\mathrm{SiC}$ induced by EDAMM was performed using both $\mathrm{AC}$ and DC discharges.

\section{Materials and methods}

The starting powder was hexagonal $\mathrm{SiC}$ obtained from Aldrich (product number 357391) with a particle size of 400 mesh and $97.5 \%$ purity. As advised by Aldrich, the $\mathrm{SiC}$ was synthesized by high temperature reduction of silica with coke (salt was used as flux) in an electric furnace. EDAMM was performed in a modified vibratory laboratory rod mill. The mill 
was designed to produce a milling mode combining a repeated impact of a curved stainless steel rod end on powder particles, placed on a vibrating hemispherical stainless steel container under electrical conditions of pulsed discharges (Figure 1). During milling, pulsed discharges are initiated at the tip of the rod, travel through the milling atmosphere and powders, terminating in the base at the centre of the hemispherical milling cell. The power supply unit is custom built specifically for discharge milling, yielding a discharge current of 3.12A with the averaged voltage of $100 \mathrm{~V}$ for alternating current and a peak voltage of $600 \mathrm{~V}$ for the direct current. It should be noted that no phase transformation occurs with the discharge switched off. More specific information is given in Table 1 with comparison to the traditional planetary ball milling.

Fig. 2 shows oscilloscope voltage vs time images of the discharges. In AC mode the detected pulsed waveform (high voltage low current) is relative to a base of zero voltage and current while in DC mode the pulsed waveform is applied on top of a negative discharge voltage and constant current. The EDAMM experiments were carried out under atmospheric pressure of nitrogen, using AC and DC milling modes. The average gap between electrodes was $6 \mathrm{~mm}$. It has to be born in mind that the experimental procedure and conditions of processing were both based on the fact that no phase transformation was occurring when the discharge was switched off and so the impact on the particles within the same time frame is not causing any chemical reactions or diffusion on the atoms.

X-ray diffraction analysis of starting and milled powders was performed using a Philips Diffractometer with $\mathrm{Cu} \mathrm{K} \alpha$ radiation and graphite monochromator. Diffractometer output was analysed using Traces Version 5.1.0 software. Phase identification was carried out using the International Centre for Diffraction Data (JCPDS-ICDD 2000) Powder Diffraction Files (PDF). Powder morphologies were characterized using a JOEL 7001 FEGSEM scanning electron microscope (SEM). Elemental composition was also performed using energy dispersive spectroscopy (EDS) using a Bruker EDS detection system running in semi-quantitative standardless analysis mode. Transmission electron microscopy (TEM) was performed using a JEOL $2011200 \mathrm{KeV}$ high resolution analytical instrument. TEM samples were prepared by dispersion of $\mathrm{SiC}$ powders onto holey carbon support grids. Polished powder samples were prepared by hot mounting machine under $4200 \mathrm{kPa}$ at $150{ }^{\circ} \mathrm{C}$ for 8 minutes. The nanohardness measurements were carried out by UMIS nanoindenter and the Vickers hardness test was performed with hardness tester machine LECO M-400-H1 with 50g load imposed for 5 sec. An average value gained from the measurements was taken from 8 indentations.

\section{Results and discussion}

Following a series of trials during which the applied power during EDAMM processing was varied the highest reaction rates of the as-received powders were found when the averaged discharge power was around $0.54 \mathrm{~kW}$. Higher discharge powers, of up to $3 \mathrm{~kW}$, were found to cause excessive powder particles agglomeration which generally slows down transformation rates.

\subsection{Phase identification.}

There are more than 250 different polymorphic crystal structures of $\mathrm{SiC}$ whose electromagnetic properties may differ significantly. Within that group we can distinguish socalled polytypes which are characterized by a unique shift in the bilayer lattice between the $\mathrm{SiC}$ unit cells otherwise called bilayer stacking. Because of the relatively easier manufacturing of a single type $\mathrm{SiC} 4 \mathrm{H}, 6 \mathrm{H}$ and $3 \mathrm{C}$ types are of the most interest. In case of a $3 \mathrm{C}$ cubic and the $4 \mathrm{H}$ and $6 \mathrm{H}$ hexagonal structures, differences in stacking sequence do not alter the distance between those atom planes which give rise to the most intense lines of the $\mathrm{x}$-ray diffraction patterns. For 
this reason it can be difficult to distinguish between polytypes on the basis of XRD results alone [17], when a mixture of cubic and hexagonal phases are present. Therefore in this study we combined x-ray analysis with TEM and electron diffraction.

X-ray diffraction revealed structures present in the starting powder (Fig. 3(a)) to be consistent with a mixture of the hexagonal 4H structure (PDF Card, 02-1463; $\mathrm{a}=0.30730 \mathrm{~nm}$ and $\mathrm{c}=1.0053 \mathrm{~nm}$ ) and the hexagonal $6 \mathrm{H} \alpha$ structure (PDF Card 42-1360; $\mathrm{a}=0.30730 \mathrm{~nm}$ and $\mathrm{c}$ $=1.511 \mathrm{~nm})$. Starting powders were processed under EDAMM for 5 and $10 \mathrm{~min}$ in both DC and AC modes to observe effects of processing parameters on product formation. In Fig. 3 X-ray diffraction patterns obtained from samples after EDAMM for 5 and $10 \mathrm{~min}$ in DC mode are presented. Note that XRD peaks related to the Hexagonal SiC phases alone show much lower intensities and only those hexagonal $\mathrm{SiC}$ peaks, which overlap with the 3C-SiC, show the same or higher intensity, a result consistent with the formation of a significant proportion of 3C-SiC. XRD results from the sample after 10 min of EDAMM show a set of peaks that can be indexed to 3C-SiC (PDF 02-1050; $\mathrm{a}=0.4349 \mathrm{~nm}$ ). Fig. 4(b) and (c) show x-ray diffraction patterns obtained from samples after EDAMM for 5 and 10 min in AC mode. In this case a full set of 3CSiC peaks is seen after 5 min of EDAMM (Fig. 3(b)). Prolonged milling for 10 min results in formation of additional peaks corresponding to a non-stoichiometric $\mathrm{Fe}_{0,42} \mathrm{Si}_{2,67}$ phase (PDF 41122). This phase is believed to have formed as a result of a processing induced reaction between the steel (plunger and milling base) and silicon after partial decomposition of the SiC.

Transmission electron microscopy was used to investigate the morphology and structure of EDAMM processed samples. Fig. 5 shows TEM results obtained from the sample processed for 10 mins under DC mode. The nanostructural morphology of the agglomerates formed was confirmed, with a mixed nanocrystalline size, as indicated in (Fig. 5(a)). The 3C-SiC cubic structure of the sample was confirmed by electron diffraction of the selected area (Fig. 5(b)), while dark field imaging (Fig 5(c)) confirmed that the nanostructural particles were distributed throughout the particle's agglomerates. In contrast to the EDAMM processed samples, the starting powder was coarse. Fig. 5(d) shows a starting powder particle, the top end producing HRTEM contrast consistent with that of a faulted hexagonal SiC phase.

On the basis of the XRD and TEM results we can conclude that the rate of $3 \mathrm{C}$-SiC formation under AC mode discharge milling is faster than under DC mode. This phenomenon possibly relates to the different kinetic conditions during EDAMM, and the transport of particles to the centre of the milling cell. It has been established from previous investigations that under DC mode processing localized Joule heating of particles occurs in the vicinity of the electrical discharges as they pass through the powder. This would imply that the hexagonal SiC particles at the centre of the cell experience a higher maximum temperature than those located at the edges of the cell. On the other hand, under AC mode the discharge is less localized and covers much wider area, thus the particles at the centre and at the edges are more equally heated to a maximum temperature where reaction is most rapid. The mechanical milling action of the stainless steel rod facilitates mixing during EDAMM and combined with the more even temperature distribution associated with milling for 5 minutes in AC mode results in an overall effect, where more particles experience the maximum temperature under AC mode consistent with the observed increase of the transformation rate compared to DC mode.

As seen in the experimental work above, the reverse transformation occurs both in the $\mathrm{AC}$ and DC plasma in Nitrogen atmosphere. It should be noted that $\mathrm{N}_{2}$ plays an important role as an energy carrier which in comparison to Argon is a diatomic gas subjected to first dissociation and ionization. Because of that reason, $\mathrm{N}_{2}$ is able to supply much higher energy content in a 
lower temperature than monoatomic gases and force the thermodynamically unfavorable transformation.

The speed of any phase formation is related to the type of the transformation process like diffusional or interface. In processes like grain growth that do not involve a composition change but only interface motion, the boundary migration kinetics involve local atomic rearrangements as atoms jump from one grain to their neighbour. Such process is generally considered as interface controlled. Often, interface-controlled motion occurs at constant velocity and it is found to be linear in time.

\subsection{Size and morphology trends with processing and Hardness Measurements}

Fig. 6 compares SEM images obtained from the hexagonal SiC starting powder; (a) with products formed after discharge milling in nitrogen under AC mode (Fig 6 (b) and (c)) and DC mode (Fig. 6(d-f)). The SiC starting powder (Fig. 6(a)) comprised large irregular particles with diameters ranging in between 20-40 $\mu \mathrm{m}$ and a fraction of smaller particles below $20 \mu \mathrm{m}$. After 5 minutes of discharge milling under AC mode (Fig. 6(b)) the average particle size is reduced and agglomerations of small particles are present. During the first 5 minutes of milling the relatively large powder particles undergo fragmentation, presumably caused by ion surface bombardment and mechanical action of the plunger, while very small powder particles produced are believed to agglomerate under the influence of strong electrostatic forces. Fig. 6(c) shows the powder morphology after 10 minutes of milling, which comprises predominately clumps of $\sim 1-5 \mu \mathrm{m}$ in size, of very small particles which have agglomerated. Similar trends in particle size reduction and agglomeration were observed in the sample milled under DC mode for 5 (Fig. 6(d)) and 10 min (Fig. 6(e)), with indication of a less uniform particle size distribution in the samples milled for $10 \mathrm{~min}$.

As mentioned in at the start of this Section, the usage of discharge power up to $3 \mathrm{~kW}$ causes excessive powder particles agglomeration. This phenomenon can be applied to form fully sintered, high density particles of sufficient size to perform nanohardness test on, for example, the particle shown in Fig. 6(f). To achieve such morphology the EDAMM process is performed in two stages where; (i) low power EDAMM, up to $0.54 \mathrm{~kW}$, is used for up to 10 min to allow transformation from hexagonal to cubic $\mathrm{SiC}$ and then (ii) additional EDAMM is performed for about 2 min, with $3 \mathrm{~kW}$ power to form large particles. Nanoindentation test was performed yielding the value of $24.4 \mathrm{GPa}$ with the standard deviation of $4 \mathrm{GPa}$ which, according to the literature [16], should give a value higher than the standard Vickers test by 10-30\%. Additionally, the measurement enabled a Young Modulus estimate. A wide range of SiC Young's moduli are reported in the literature. The highest value was reported in Gmelin's Handbook, 8th edition (1959), which listed $748 \mathrm{GPa}$ for cubic-SiC in the [100] set of directions. While directionality of polytype is not expected to affect the number greatly, defects are, and annealing and other treatments can produce widely different results for the same specimen. Later versions of Gmelin's (1984) [18] report values of Young's modulus in the range of 400-500 $\mathrm{GPa}$. On the other hand, [19] reported a value of $302.8 \mathrm{GPa}$ while in [19] the reported value is $240 \mathrm{GPa}$. In our samples the Young's modulus value was found to be $209.6 \mathrm{GPa}$ which is a typical one for the recrystallized state of commercially produced $\mathrm{SiC}$ [21]. During extended EDAMM processing powders are subjected to localized intensively heated areas by the formation of the electric discharge. This condition is believed to be favorable for formation of microstructures similar to those associated with recrystallized states.

4. Conclusions 
Hexagonal Silicon Carbide was transformed into to cubic form by application of the Electric Discharge Assisted Mechanical Milling (EDAMM) in a nitrogen atmosphere. It was found that the transformation takes place in as little as $5 \mathrm{~min}$ of milling under the AC mode and 10 min using the DC discharges what is a result of the different heat distribution during the process. Prolonged milling times of $10 \mathrm{~min}$ in AC mode and above result in significant increase in contamination from the milling media, and formation of Iron Sillicon phase.

It was proven, in comparison to the previous research, that it indeed is possible to transform the Hexagonal $\mathrm{SiC}$ into its Cubic polytype without the additives such as $\mathrm{Al}_{2} \mathrm{O}_{3}, \mathrm{AlN}$ or $\mathrm{Si}_{3} \mathrm{~N}_{4}$ to facilitate the reaction and stabilize the cubic structure. Thus, not only is the method onestep, quick and effective but it also gives a new insight on the way the transformation takes place. Above results suggest that in the case of EDAMM, the transformation is related to the heat distribution through the particles being possibly facilitated by the Nitrogen atmosphere.

\section{Acknowledgements}

This work was supported by funding from the Australian Research Council Discovery Grant no DP130101390. Electron microscopy equipment used was funded by Australian Research Council grants LE 0882813 and LE 0237478 and located at the UOW Electron Microscopy Centre.

\section{References}

[1] B. Ghosh, S.K. Pradhan (2009) J. Alloys Compd, 486: 480-485.

[2] M. Ohyanagi, T. Yamamoto, H. Kitaura, Y. Kodera, T. Ishii, Z.A. Munir (2004) Scripta Mater 50:111-114.

[3] J.Z. Guo, Y. Zuo, Z.J. Li, W.D. Gao, J.L. Zhang (2007) Physica E, 39: 262-266.

[4] H.-P. Martin, R. Ecke, E. Müller (1998) J. Eur. Ceram. Soc. 18:1737-1742.

[5] R. Koc, S. Cattamanchi (1998) J. Mater. Sci. 33: 2537-2549.

[6] F.K. van Dijen, R. Metselaar (1991) J. Eur. Ceram. Soc. 7:177-184.

[7] C. Czosnek, M.M. Bucko, J.F. Janic et al. (2015) Mater. Res. Bull. 63:164-172.

[8] H. Zhuang, C. Wang, N. Huang and X. Jiang, (2014) Electrochem. Commun. 41: 5-7.

[9] L. G. Hanscomfield (1967) Physical Sciences Research Papers, 336, Bedford, Massachusetts

[10] S. Turan, K. Knowles (1996) J. Am. Ceram. Soc. 79: 2892-96

[11] A.L.Ortiz, A. Munoz-Bernabe, O. Borrero-Lopez, A.Dominguez-Rodriguez, F. Guiberteau (2004) J. Am. Ceram. Soc. 24: 3245-3249

[12] B.Ghosh, S.K. Pradhan (2009) J. Alloys Compd. 486:480-485

[13] A.Lara, A. L. Ortiz, A. Munoz, A. Dominguez-Rodriguez (2012) Ceram. Int., 38: 45-53.

[14] C. Ragaru, M. Lancin, C. Marhic (1999) J. Eur. Ceram. Soc. 19: 2701-2709.

[15] A. Calka, D. Wexler (2002) Nature, 419: 147-151.

[16]http://www.retsch.com/products/milling/ball-mills/planetary-ball-mill-pm-100/functionfeatures/

[17] A.L. Ortiz, F. Sánchez-Bajo, F.L. Cumbrera, F. Guiberteau, (2001) Mat. Lett. 49: 137-145

[18] Gmelin, Handbook of Inorganic and Organometallic Chemistry edited by G. Kirschstein and D. Koschel (1984) Silicon, Springer

[19] J. F. Shackelford, W. Alexander (2000) CRC Materials Science and Engineering Handbook $3^{\text {rd }}$ Edition, CRC Press

[20] M.A. El Khakani, M. Chaker, A. Jean, S. Boily, J.C. Kieffer, M.E. O'Hern, M.F. Ravet, F. Rousseaux (1994) J. Mater. Res, 9: 96-103.

[21] http://www.alphamaterials.com/R_SiC.htm 
Table 1 Standard Ball Milling Device specifications comparison with the EDAMM device.

\begin{tabular}{lll}
\hline Specification & Retsch and Fritsch planetary mill [16] & EDAMM \\
\hline Discharge current & - & $3.12 \mathrm{~A}$ \\
\hline Averaged voltage & - & $100 \mathrm{~V}$ \\
\hline Max voltage in peak & - & $600 \mathrm{~V}$ \\
\hline Impact force & $33.3 \mathrm{~g}(\mathrm{G}$ force on ball) & $0.04 \mathrm{~N}$ \\
\hline Velocity before impact & $4.427 \mathrm{~m} / \mathrm{s}$ & $0.313 \mathrm{~m} / \mathrm{s}$ \\
\hline Impact kinetic energy & $0.3234 \mathrm{~J}$ & $0.004 \mathrm{~J}$ \\
\hline
\end{tabular}

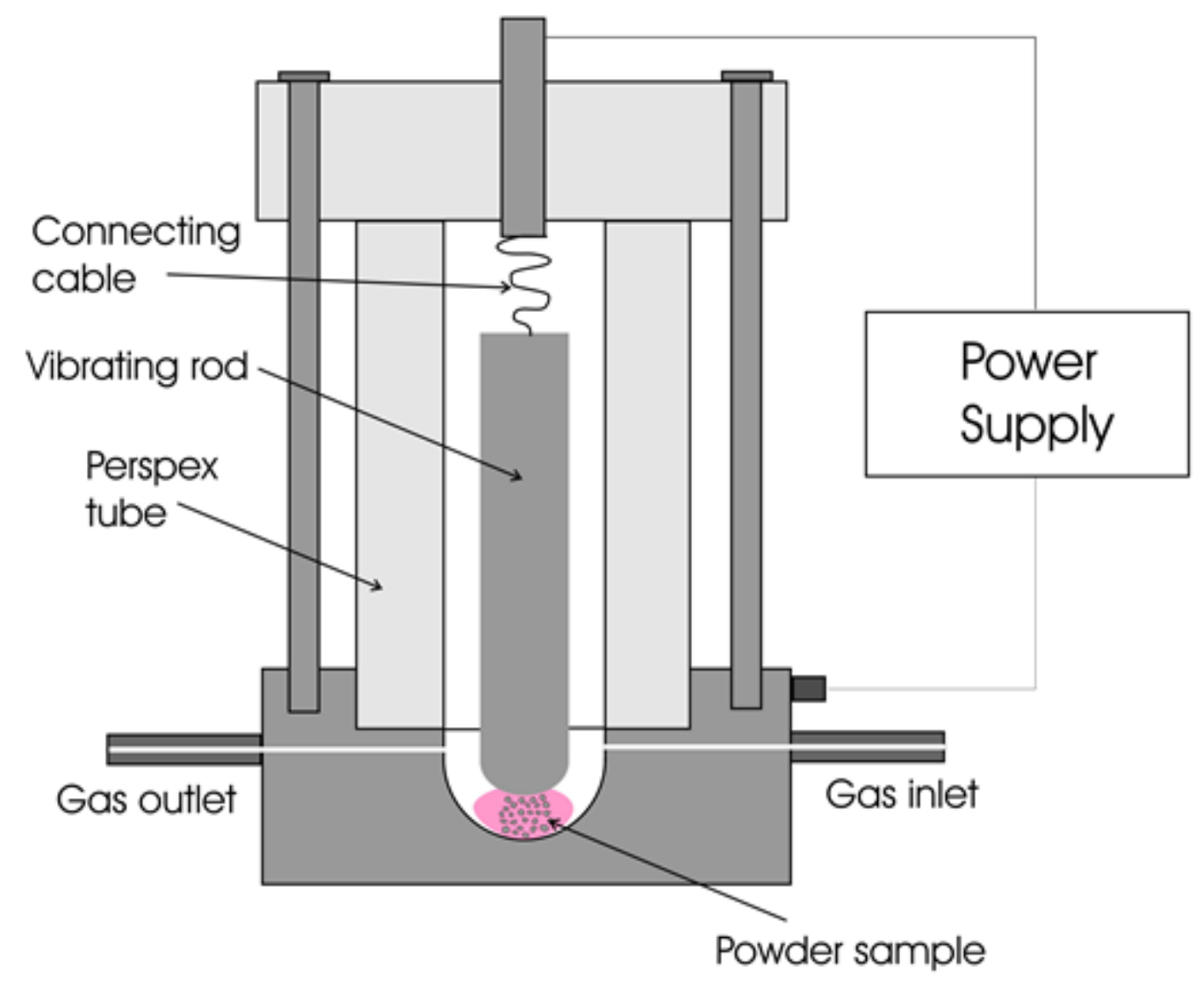

Figure 1 Schematic representation of the EDAMM method. 

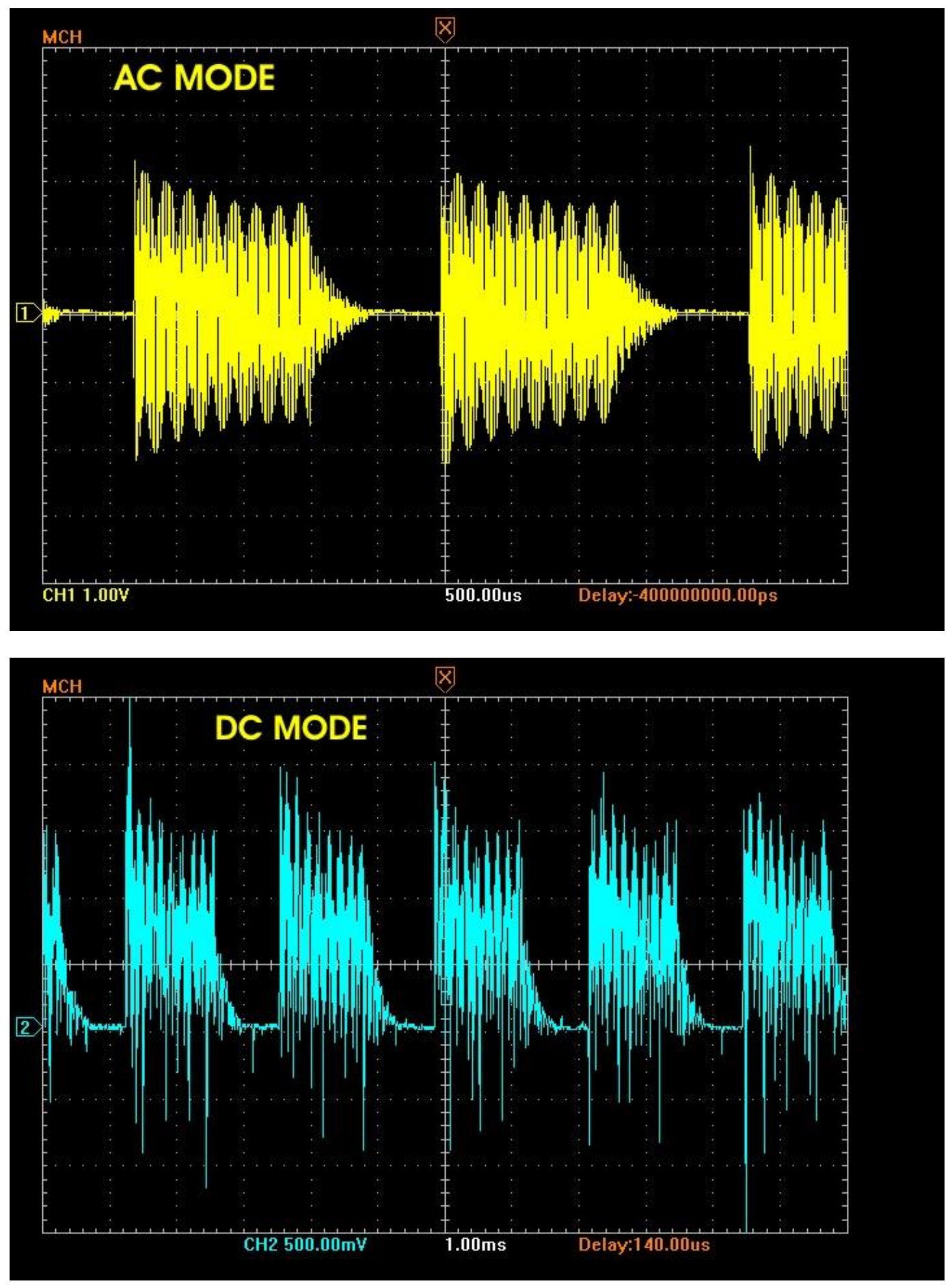

Figure 2 Oscilloscope images showing differences in high frequency AC mode (top) and lower frequency DC mode (bottom) pulsed electrical discharges, as detected during EDAMM processing. 


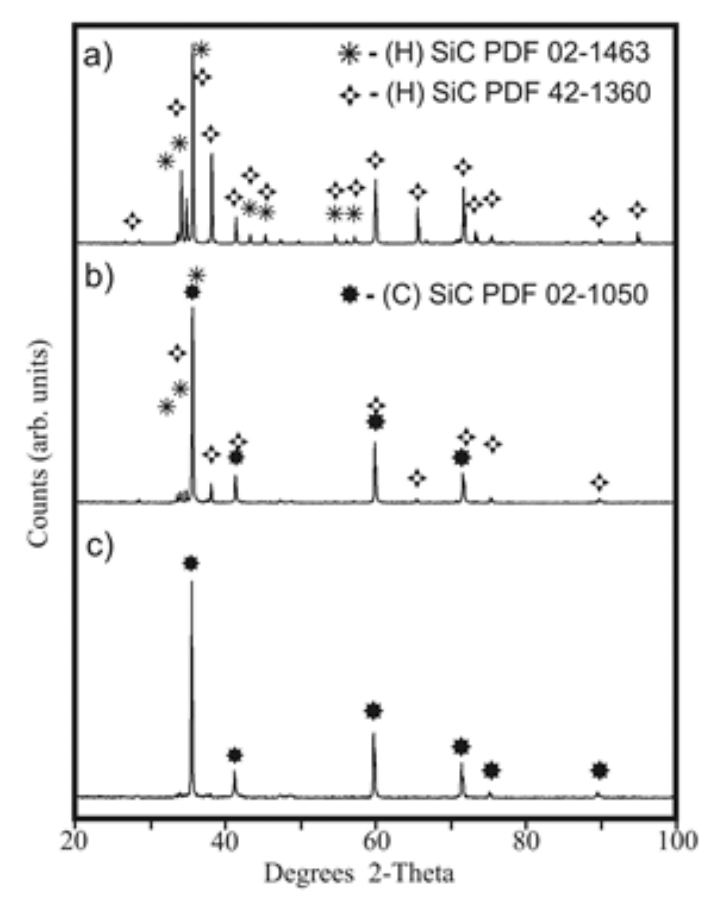

Figure 3 XRD of a) starting powders and powders processed in Nitrogen by EDAMM for b) 5 and c) 10 min using DC mode.

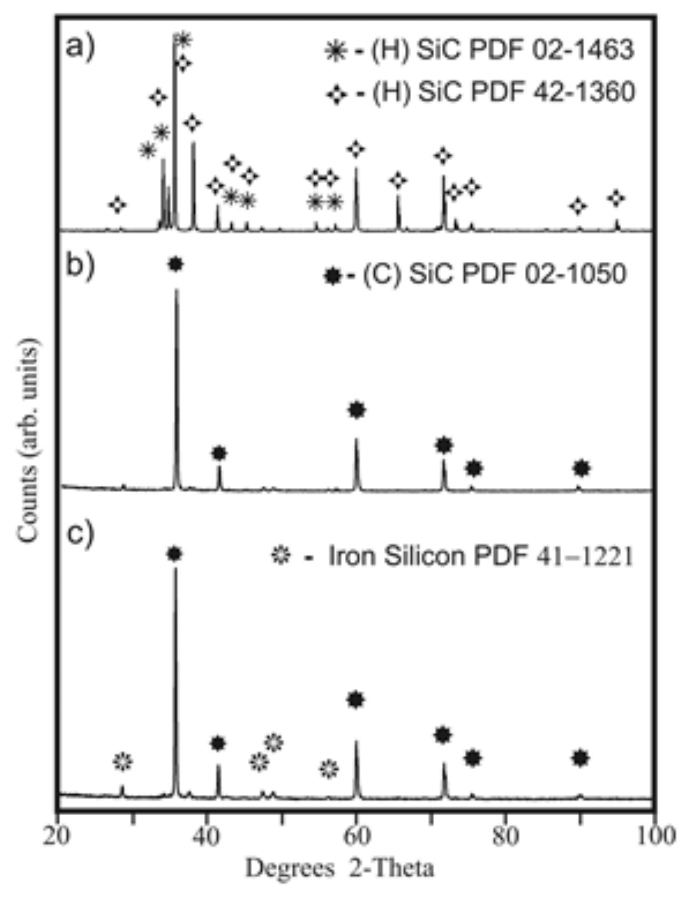

Figure 4 XRD of a) starting powders and powders processed in Nitrogen by EDAMM for b) 5 and c) 10 min using AC mode. 

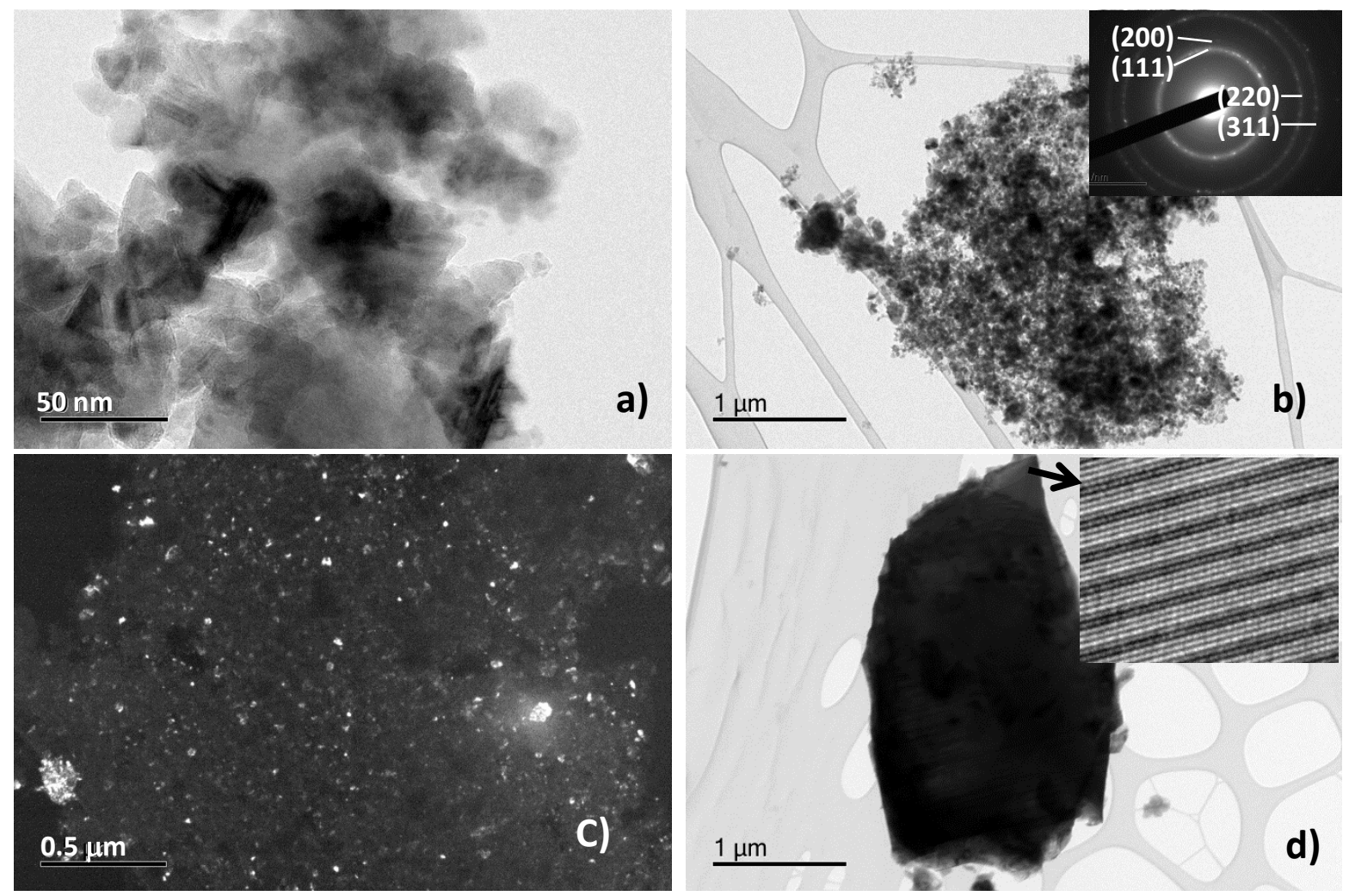

Figure 5 TEM Observations: (a)-(c) Sample EDAMM processed for 10 mins under DC mode. Bright field image (a) shows variation in crystallite size. Inset diffraction associated with region shown in (b) is indexed according to cubic $\mathrm{SiC}$, (c) a higher magnification dark field image of (b), with aperture centered around (111) and (200) diffraction rings; structure. Image (d) is of the starting powder. HRTEM contrast from the top of this particle is consistent with a structure comprising faulted hexagonal $\mathrm{SiC}$ phases. 

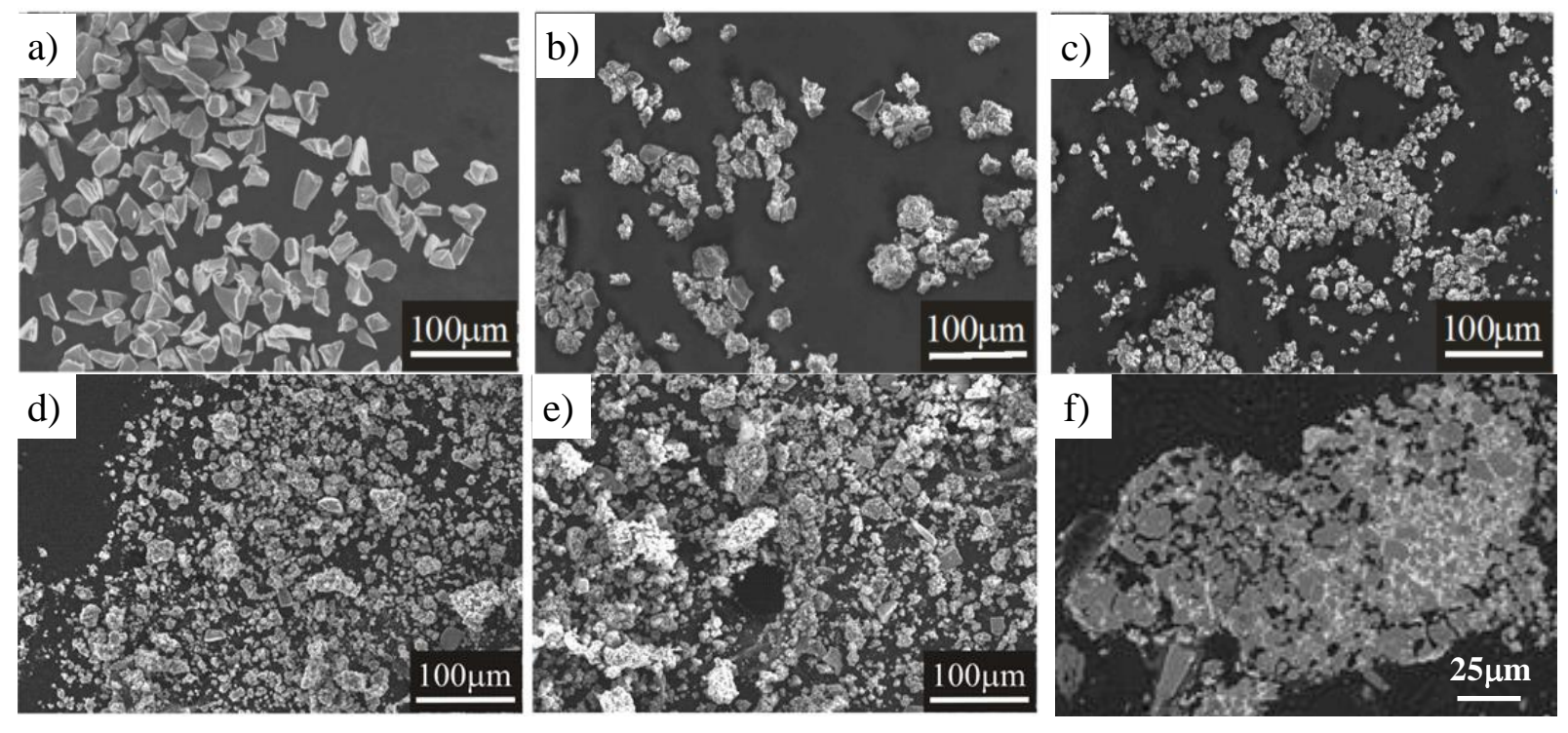

Figure 6 SEM results: Secondary electron images of (a) dispersed starting powder; product after $4 \mathrm{~kW}$ EDAMM under AC mode for (b) 5 min and (c) $10 \mathrm{~min}$; product after EDAMM under DC mode for (d) 5 min and (e) $10 \mathrm{~min}$. (f) Higher magnification backscattered image agglomerated product, polished and sectioned. The sample shown in (f) was prepared by EDAMM processing of sample (e) for an additional 2 mins at high power $(3 \mathrm{~kW})$. 